\title{
Pretargeted Imaging and Therapy
}

\author{
Mohamed Altai ${ }^{1}$, Rosemery Membreno ${ }^{2-4}$, Brendon Cook $^{2-4}$, Vladimir Tolmachev ${ }^{1}$, and Brian M. Zeglis ${ }^{2-4}$ \\ ${ }^{I}$ Department of Immunology, Genetics, and Pathology, Uppsala University, Uppsala, Sweden; ${ }^{2}$ Department of Chemistry, Hunter \\ College of the City University of New York, New York, New York; ${ }^{3}$ PhD Program in Chemistry, Graduate Center of the City University \\ of New York, New York, New York; and ${ }^{4}$ Department of Radiology, Memorial Sloan Kettering Cancer Center, New York, New York
}

In vivo pretargeting stands as a promising approach to harnessing the exquisite tumor-targeting properties of antibodies for nuclear imaging and therapy while simultaneously skirting their pharmacokinetic limitations. The core premise of pretargeting lies in administering the targeting vector and radioisotope separately and having the 2 components combine within the body. In this manner, pretargeting strategies decrease the circulation time of the radioactivity, reduce the uptake of the radionuclide in healthy nontarget tissues, and facilitate the use of short-lived radionuclides that would otherwise be incompatible with antibody-based vectors. In this short review, we seek to provide a brief yet informative survey of the 4 preeminent mechanistic approaches to pretargeting, strategies predicated on streptavidin and biotin, bispecific antibodies, complementary oligonucleotides, and bioorthogonal click chemistry.

Key Words: pretargeting; multistep targeting; click chemistry; bispecific antibody; streptavidin; biotin

J Nucl Med 2017; 58:1553-1559

DOI: 10.2967/jnumed.117.189944

$\mathbf{T}$ he specificity and affinity of antibodies have long made them enticing vectors for the delivery of diagnostic and therapeutic radionuclides to malignant tissue. Yet one of the fundamental traits that makes immunoglobulins effective as agents of the immune response can spell trouble in the context of nuclear medicine. Antibodies have evolved to possess long serum half-lives, undoubtedly a benefit in the context of detecting foreign antigens. However, this means that when antibodies are harnessed as biomedical vectors, they can take days or even weeks to reach their optimal biodistribution in vivo and must therefore be labeled with radionuclides with multiday physical halflives. Although the use of isotopes such as ${ }^{89} \mathrm{Zr}$ (half-life, $3.3 \mathrm{~d}$ ) and ${ }^{177} \mathrm{Lu}$ (half-life, $6.7 \mathrm{~d}$ ) ensures that radioactivity remains after the antibody has reached its target, the choice of these nuclides is a double-edged sword. The prolonged circulation of radioimmunoconjugates bearing long-lived

\footnotetext{
Received May 11, 2017; revision accepted Jun. 27, 2017.

For correspondence or reprints contact: Brian M. Zeglis, Hunter College of the City University of New York, 413 E. 69th St., New York, NY 10021.

E-mail: bz102@hunter.cuny.edu

Published online Jul. 7, 2017.

COPYRIGHT (C) 2017 by the Society of Nuclear Medicine and Molecular Imaging.
}

radionuclides creates significant clinical complications: supobtimal therapeutic indices for radioimmunotherapy and high radiation doses to healthy tissue for antibody-based PET.

A tremendous amount of effort has been dedicated to circumventing these obstacles. One approach has centered on bioengineering lower-molecular-weight immunoglobulins with more rapid excretion rates. Yet despite the promise of this avenue, radiolabeled antibody fragments are often hampered by suboptimal tumor uptake and high retention in the kidneys. An alternative solution lies in the topic of this work: in vivo pretargeting. Conceived in 1985 and first executed 2 y later, pretargeting is founded on a simple yet radical premise: decoupling the antibody and the radioactivity $(1,2)$. The 2 components are injected separately and combine within the body, in essence performing radiosynthesis at the tumor itself. A variety of mechanistic platforms for pretargeting have been developed, yet all share 2 common components: a radioligand and an antibody capable of binding both a tumor antigen and said radioligand. Generally speaking, pretargeting strategies have 4 steps: first, the injection of the antibody; second, the slow accumulation of the antibody at the tumor and its concomitant clearance from the blood; third, the injection of the radioligand; and fourth, the in vivo ligation of the antibody and the radioligand, followed by the rapid clearance of any excess radioligand (Fig. 1A). Some methodologies feature an additional step before the injection of the radioligand, the administration of a clearing agent designed to accelerate the removal of residual immunoconjugate from the bloodstream. Furthermore, conventional wisdom dictates that vectors for pretargeting should not be internalized upon binding their target, though a handful of recent investigations indicates that pretargeting is possible with internalizing systems. Details aside, injecting the radionuclide and immunoglobulin separately decreases the circulation time of radioactivity in the body, reduces the uptake of the radioisotope in healthy tissues, and facilitates the use of short-lived radionuclides (e.g., ${ }^{68} \mathrm{Ga}$ ) that would normally be incompatible with antibodybased vectors. Taken together, these traits translate to improved tumor-to-background activity concentration ratios and dramatically lowered radiation dose rates to healthy tissues.

The central problem at the core of any pretargeting strategy lies in how to recombine the antibody and radioligand in vivo. The interaction between the 2 components 


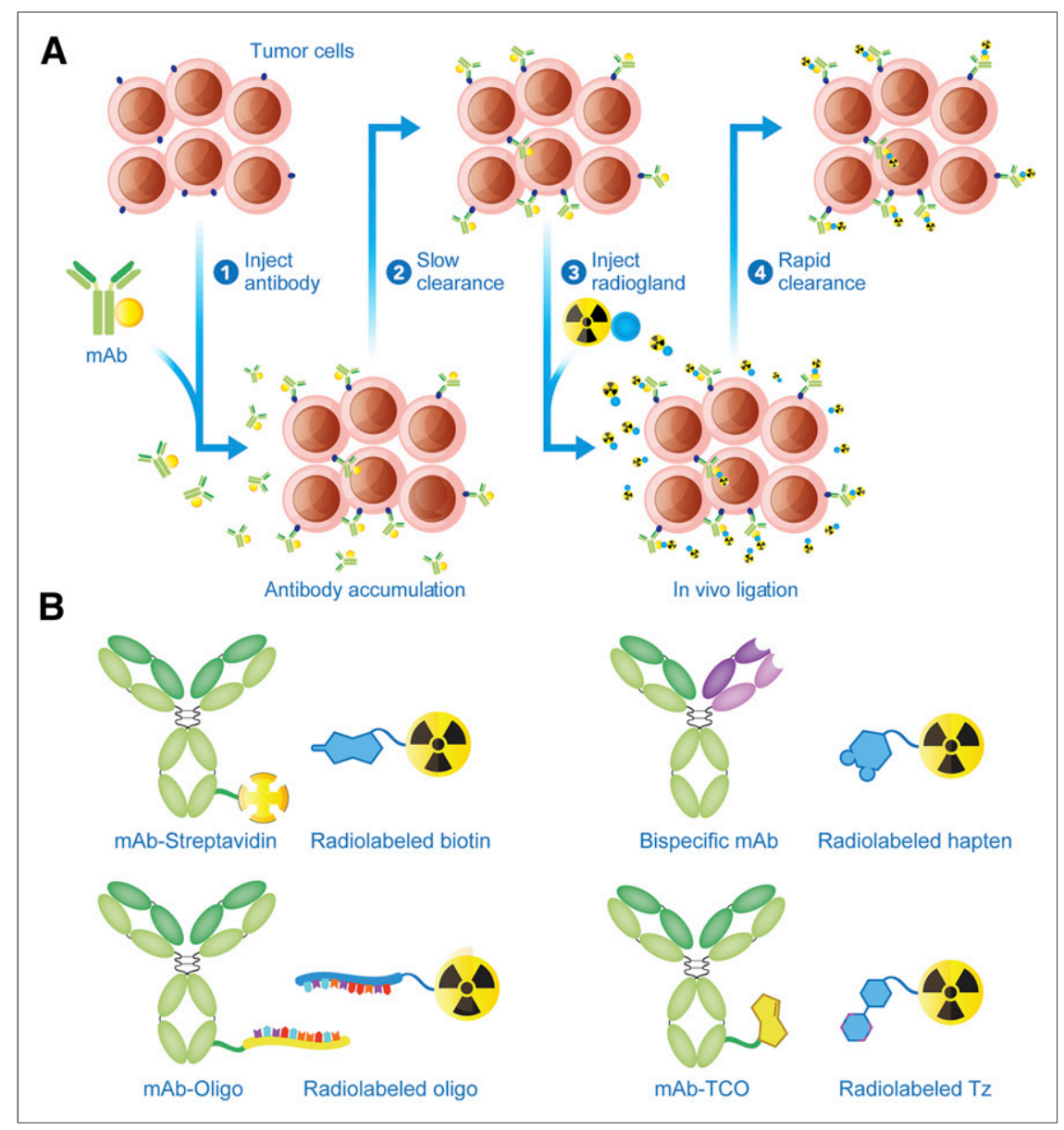

FIGURE 1. Schematic for in vivo pretargeting $(A)$ and the 4 principal mechanisms of in vivo pretargeting $(\mathrm{B})$.

must be exquisitely selective, as each moiety has myriad other possible reaction partners within the body. Over the years, 4 major approaches have emerged. Each relies on a different in vivo ligation mechanism: the noncovalent interaction between streptavidin and biotin, the ability of bispecific antibodies (bsAbs) to bind both an antigen and a radiolabeled hapten, the hybridization of complementary oligonucleotides, and the bioorthogonal inverse electron demand Diels-Alder (IEDDA) click reaction (Fig. 1B). In this review, our goal is to provide a brief introduction to these approaches. Critically, we do not strive for an exhaustive treatment of any of these methods, as others have already done this with impressive depth and clarity $(3,4)$. Rather, our mission is to provide a bird's-eye view meant for experienced researchers and novices alike, in the hope that this primer will not only spur enthusiasm for pretargeting but also inspire innovation that will drive the field in the years to come.

\section{APPROACH 1: STREPTAVIDIN AND BIOTIN}

Pretargeting approaches based on the noncovalent interaction between biotin and streptavidin were among the earliest strategies to emerge and the first to appear in the clinic. Biotin is a $244-\mathrm{Da}$ small molecule and an essential coenzyme for carboxylases (Fig. 2A). Streptavidin and avidin are tetrameric proteins composed of 4 monomers, each capable of binding a single biotin (Fig. 2B). The noncovalent interactions between biotin and these proteins are among the strongest observed in the natural world, with binding constants approaching $10^{15} \mathrm{M}^{-1}$. Not surprisingly, both avidin and streptavidin have been leveraged for pretargeting; however, our discussion here will focus primarily on streptavidin, as it has been the subject of wider use in clinical trials $(2,5)$.

Three different approaches to biotin and streptavidin pretargeting have been developed. In the first, a biotin-bearing antibody and a radiolabeled streptavidin are used (Fig. 2C) (6). This approach has been largely abandoned, however, as the protracted circulation times of the radiolabeled tetramer undermine the fundamental premise of pretargeting. The second strategy uses a biotin-based radioligand and a streptavidin-bearing immunoconjugate. In this case, the latter can be made via the creation of a fusion protein, the chemical conjugation of streptavidin to an antibody, or the noncovalent conjugation of streptavidin to a biotin-bearing antibody (Fig. 2D) (7). A significant obstacle to this approach is the presence of endogenous biotin in the blood. This biotin threatens to saturate the binding sites of the antibody-bound streptavidin, prompting the use of a clearing agent to remove excess biotin from circulation. A third technique-a 3-step strategy called avidin bridging-was also devised to combat natural biotin (Fig. 2E) (5). Here, a biotinylated antibody is administered first, followed later by avidin or streptavidin. The protein simultaneously serves 2 purposes: clearing the blood of endogenous biotin and binding to the monoclonal antibody-biotin construct at the target site. Once the free avidin or streptavidin clears, a biotin-based radioligand is injected that can then bind to 1 of the 3 remaining sites of the antibody-bound protein at the target site.

Streptavidin and biotin-based pretargeting systems have proven enormously successful in preclinical studies. These approaches have been used with radionuclides ranging from ${ }^{90} \mathrm{Y}$ to ${ }^{186} \mathrm{Re}$, yielding impressive tumoral uptake while simultaneously lowering both activity concentrations in 


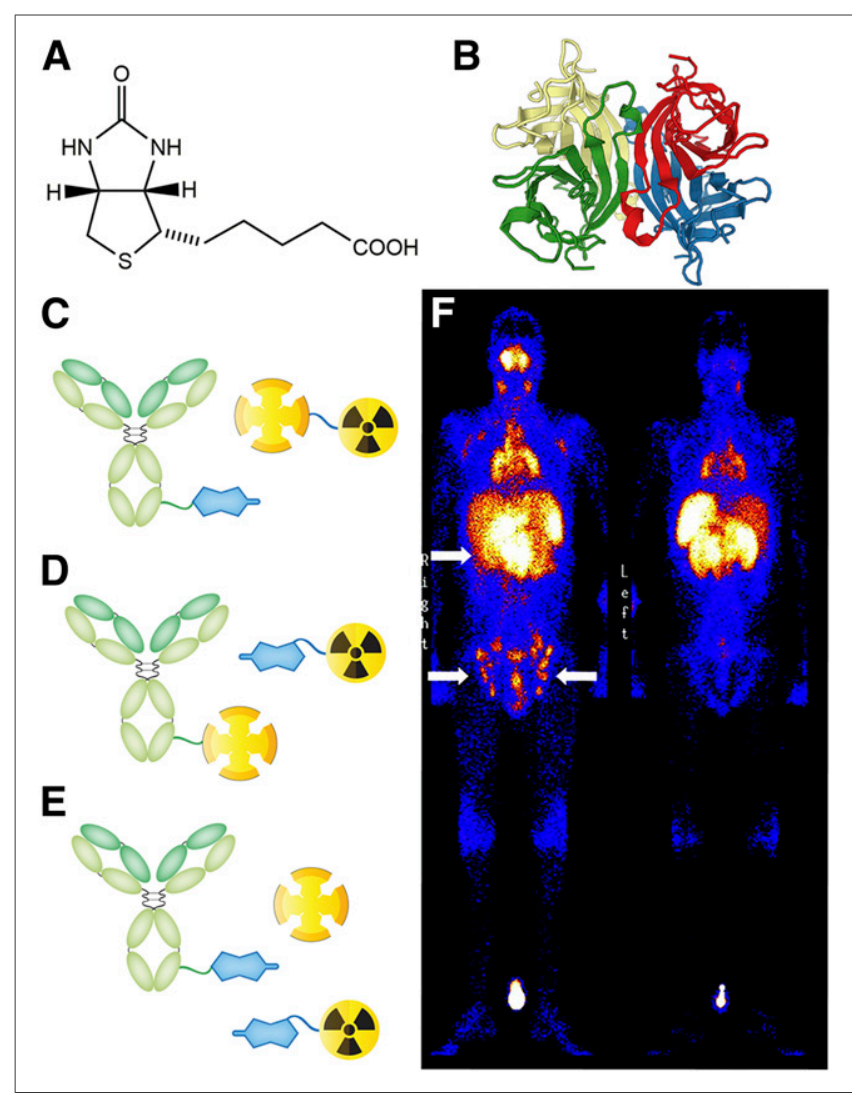

FIGURE 2. Structure of biotin (A); ribbon structure of the streptavidin tetramer (B); 3 different types of streptavidinbiotin pretargeting strategies (C-E); and $\gamma$-camera image of a patient with B-cell Hodgkin lymphoma injected with an antiCD20-SA fusion protein and, $24 \mathrm{~h}$ later, ${ }^{111} \mathrm{In}$-DOTA-biotin (F). Sites of active tumor involvement are indicated by arrows. (Reprinted with permission of (10).)

the blood and overall effective dose rates. In light of these data, several phase I and II clinical trials were initiated to probe efficacy in humans (7-9). Initial results using a monoclonal antibody-streptavidin/radiolabeled-biotin methodology proved promising. One phase II study of pretargeted radioimmunotherapy (PRIT) using ${ }^{90} \mathrm{Y}$ showed that patients with non-Hodgkin lymphoma were able to withstand a higher ${ }^{90} \mathrm{Y}$ dose during PRIT than during traditional radioimmunotherapy (8). Another trial in high-grade glioma patients demonstrated an increased median survival time for PRIT patients compared with untreated controls (9). Furthermore, Forero et al. demonstrated the clinical feasibility of pretargeted SPECT, using a monoclonal antibody-streptavidin conjugate and an ${ }^{111}$ In-biotin radioligand (Fig. 2F) (10).

Despite these successes, a critical observation in all of these studies was that patients administered streptavidinbased immunoconjugates exhibited increased levels of human anti-streptavidin antibody $(7,8,10)$. Once a patient begins to express high enough levels of human antistreptavidin antibody, subsequent treatments can trigger an allergic reaction that leads to severe treatment-associated morbidity. This is particularly relevant in PRIT, as the administration of fractionated doses is common. Unfortunately, this immunogenicity has proven to be a nearly fatal flaw. Streptavidin-based pretargeting has received little attention for nearly a decade, and most of the field's momentum has shifted elsewhere.

\section{APPROACH 2: BISPECIFIC ANTIBODIES}

A second approach to in vivo pretargeting relies on bsAbs engineered to bind both a tumor-associated antigen and a radiolabeled hapten (4). Over the years, 3 different systems for pretargeting based on bsAbs have been developed. The first uses immunoconjugates bearing 2 different targetbinding domains: one specific to a tumor-associated antigen and another specific to a radiolabeled hapten. These include conjugates in which an antigen-binding $\mathrm{Fab}^{\prime}$ is chemically coupled to a hapten-binding Fab' as well as more sophisticated $\mathrm{IgG}$-single-chain variable fragment $(\mathrm{ScFv})$ fusion constructs bearing 2 antigen-binding Fab' domains alongside a pair of hapten-binding ScFvs (Figs. 3A and 3C). Most of this work has used haptens based on radiometal chelate complexes, including a particularly interesting recent study demonstrating PRIT in mice (11). One concern with this approach, however, is that the affinity of the radioligandspecific Fab' is highly sensitive to the coordination properties of the radiometal chelate, a trait that curtails the modularity of the system (12). This issue can be circumvented through the use of more modular peptide-based haptens such as histaminesuccinyl-glycine (HSG) (13).

A second variant of this approach also uses bsAbs bearing 2 different $\mathrm{Fab}^{\prime}$ fragments but uses a bivalent radioligand to drive tumoral uptake (Fig. 3B) (14). Bivalent haptens can bind to 2 bsAbs occupying adjacent antigens on the tumor surface, thereby cross-linking the immunoconjugates and increasing the avidity of binding. This approach yields a marked improvement compared with systems using monovalent radioligands and, in most cases, has not required a clearing agent. Boerman et al., for example, used a bivalent radioligand approach to produce tumoral activity concentrations of approximately $90 \%$ of the injected dose per gram $(\% \mathrm{ID} / \mathrm{g})$ and tumor-to-blood activity concentration ratios of approximately 148 in mice bearing CAIX-expressing xenografts (15). More recently, Kraeber-Bodéré et al. have demonstrated the clinical safety and efficacy of in vivo pretargeting using an anticarcinoembryonic antigen bsAb and a ${ }^{131}$ I-labeled bivalent hapten $(14,16)$.

A third — and arguably most promising — approach uses bsAbs bearing 2 target-specific Fab' fragments and $1 \mathrm{Fab}^{\prime}$ capable of binding an HSG hapten (Fig. 3D). The 3 components of these immunoconjugates (dubbed Tri-Fab bsAbs) are assembled and locked into place via disulfide linkages (17). HSG-based haptens dramatically increase the modularity of the system. Not only can both mono- and divalent variants be synthesized, but also a wide array of chelators and prosthetic groups can be appended to the HSG core without abrogating binding to its Fab'. Over the last $5 \mathrm{y}$, a Tri-Fab bsAb system targeting the pancarcinoma 


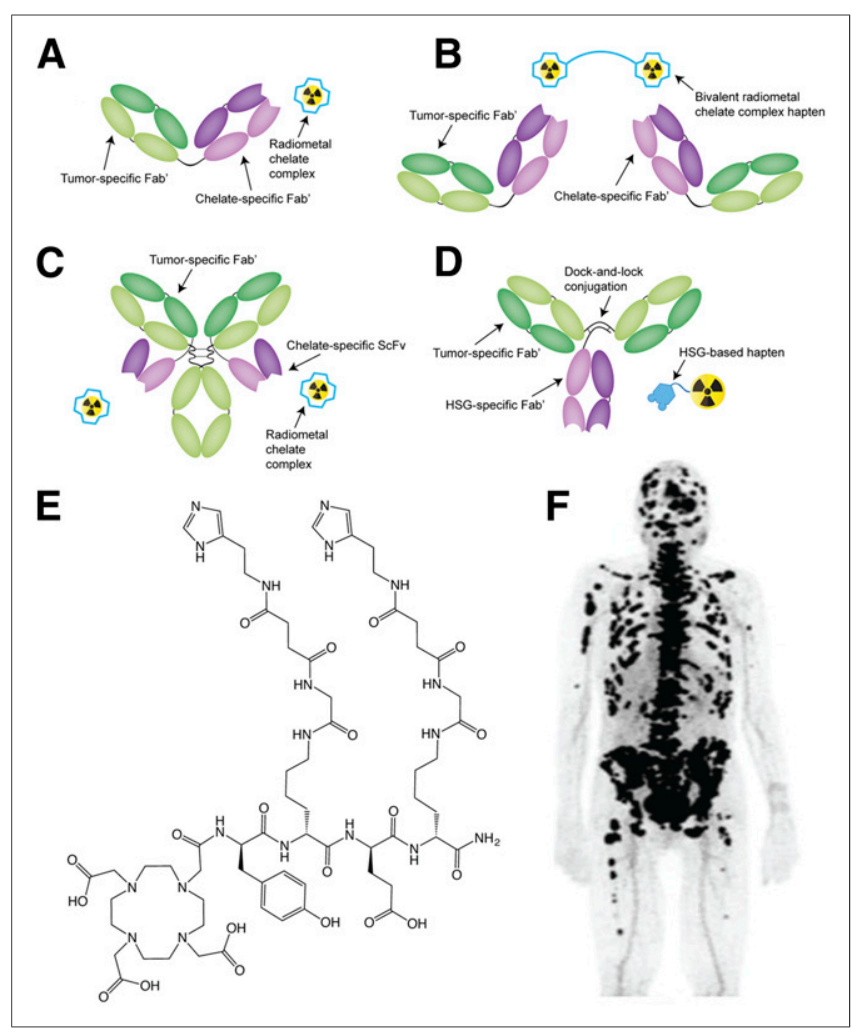

FIGURE 3. Schematics of in vivo pretargeting strategies based on chemically linked Fab' fragments (A), a divalent radiometal chelate hapten $(\mathrm{B})$, an IgG-single-chain variable fragment (ScFv) construct (C), and an HSG-binding Tri-Fab (D); structure of the divalent IMP288 HSG hapten (E); pretargeted immuno-PET image of a patient with metastatic breast cancer recorded after the administration of $120 \mathrm{nmol}$ of TF2 and, $30 \mathrm{~h}$ later, $3 \mathrm{nmol}$ of ${ }^{68} \mathrm{Ga}-\mathrm{IMP} 288(\mathrm{~F})$. (Adapted and reprinted with permission of (22).)

glycoprotein antigen Trop-2 has been developed $(18,19)$. Pretargeting using the Trop-2-targeting Tri-Fab-designated TF12 - and an ${ }^{111}$ In-labeled variant of the divalent HSG hapten IMP-228 ( ${ }^{111}$ In-IMP228; Fig. 3E) produced tumoral activity concentrations of approximately $15 \% \mathrm{ID} / \mathrm{g}$ and tumorto-blood activity concentration ratios of more than 1,000 in mice bearing MDA-MB-468 xenografts (19). Subsequently, van Rij et al. demonstrated that PRIT using TF12 and ${ }^{177} \mathrm{Lu}-$ IMP228 hapten significantly improved the median survival of mice bearing PC3 prostate cancer xenografts (18). The same laboratory also demonstrated that the TF12 platform can be leveraged for PET imaging with ${ }^{68} \mathrm{Ga}$-DOTA-IMP288 as well as multimodal imaging using a hapten bearing both ${ }^{111}$ In and a near-infrared fluorophore.

Clinical evaluation of PRIT using a carcinoembryonic antigen-targeting Tri-Fab bsAb (TF2) and ${ }^{177} \mathrm{Lu}-\mathrm{IMP} 288$ has been initiated in patients with metastatic colorectal and lung cancers (20). These studies demonstrate that this approach is feasible and safe. Infusion-related reactions were mild and could be reduced by the preadministration of prophylactic antihistamines and corticosteroids, and while human antibodies against TF2 were detected in 11 of 21 patients with colorectal cancer, immunogenicity in lung cancer was appreciably lower. Furthermore, prestudy imaging using ${ }^{111}$ In-IMP288 enabled accurate prediction of bone-marrow dose, making individualized activity dosing possible. More recently, the clinical feasibility and safety of pretargeted immuno-PET using TF2 and ${ }^{68} \mathrm{Ga}-\mathrm{IMP} 288$ was demonstrated in patients with metastatic medullary thyroid carcinoma (Fig. $3 F)(21,22)$.

\section{APPROACH 3: OLIGONUCLEOTIDES}

The next strategy is predicated on an interaction that is as specific and selective as it is ubiquitous: the hybridization of complementary oligonucleotides. Yet unmodified DNA and RNA oligomers are vulnerable to degradation by nucleases, which largely precludes their use as in vivo tools. To circumvent this issue, 2 types of nuclease-resistant DNA analogs have been exploited for in vivo pretargeting: phosphorodiamidate morpholino oligomers (MORFs) and peptide nucleic acids (PNAs).

MORFs are synthetic oligomers in which a phosphorodiamidate backbone replaces the (deoxy)ribofuranose-phosphodiester linkage (Fig. 4A). MORFs are water-soluble, are resistant to endo- and exonucleases, and retain specificity for WatsonCrick base-pairing. Much of the pioneering work on MORFbased pretargeting stems from the Hnatowich Laboratory, which first published the technique in 2002 (23). In 2011, this group reported that pretargeting using a MORF-bearing variant of the anti-tumor-associated glycoprotein 72 antibody CC49 and a ${ }^{90}$ Y-labeled complementary MORF produced tumoral activity concentrations of $7.2 \pm 2.2 \% \mathrm{ID} / \mathrm{g}$ and tumorto-blood activity concentration ratios of more than 25 (24). This same work underscored the modularity of MORF-based pretargeting, as the complementary MORF could be labeled with ${ }^{90} \mathrm{Y}$-DOTA, ${ }^{99 \mathrm{~m}} \mathrm{Tc}$-mercaptoacetyltriglycine, or ${ }^{188} \mathrm{Re}-$ mercaptoacetyltriglycine without interfering with hybridization or in vivo performance. Over the years, several promising variations on this theme have emerged, including the use of bivalent complementary MORFs, MORF-based clearing agents, and MORF-bearing dendrimers for signal amplification (25). Interestingly, Liu et al. have demonstrated the efficacy of MORF-based pretargeting for $\beta$-cell imaging, producing improved target-to-normal-organ activity concentration ratios compared to traditional immuno-SPECT (Fig. 4B) (26).

In PNAs, nitrogenous bases are conjugated to a pseudopeptide backbone made up of repetitive $N$-(2-aminoethyl)glycine units connected through amide bonds (Fig. 4A). PNAs bind to complementary strands via Watson-Crick base-pairing and are thermally stable, chemically stable, nonimmunogenic, nontoxic, and resistant to digestion by both nucleases and proteases. The feasibility of PNA-mediated pretargeting was recently demonstrated using a variant of cetuximab bearing a pair of 17-mer PNAs (27). In this work, the complementary PNA strand was PEGylated, modified with a 2,2-dipicolylamine chelator, and labeled with ${ }^{99 \mathrm{~m}} \mathrm{Tc}$. 


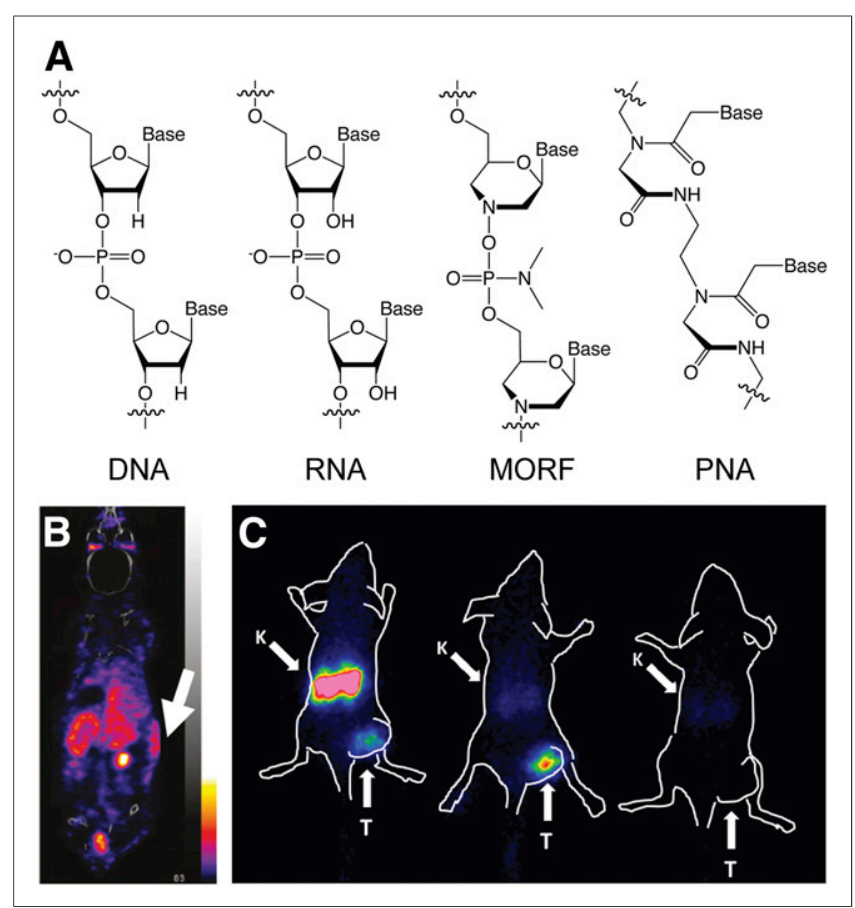

FIGURE 4. Structure of DNA, RNA, MORF, and PNA oligonucleotides (A); a pretargeted SPECT image of subcutaneous transplanted human islet cells (white arrow) in a mouse administered a MORF-modified variant of the islet-cell-specific HPi1 antibody followed by a 99mTc-labeled complementary MORF radioligand (adapted and reprinted with permission from (26)) (B); and pretargeted SPECT images of mice bearing subcutaneous SKOV3 xenografts administered a directly radiolabeled ${ }^{111}$ In-Z $Z_{\text {HER2:K58 }}$ Affibody molecule (left), a PNA-modified $\mathrm{Z}_{\text {HER2 }}$-HP1 Affibody molecule followed by complementary ${ }^{111} \mathrm{In}-$ HP2 radioligand (center), and a ${ }^{111} \mathrm{In}-\mathrm{HP} 2$ radioligand alone (right) (C). $\mathrm{K}=$ kidney; $\mathrm{T}=$ tumor (adapted and reprinted with permission from (30)).

Pretargeted SPECT experiments in mice bearing A431 xenografts revealed that tumor visualization was possible as early as $1 \mathrm{~h}$ after injection of the ${ }^{99 \mathrm{~m} T c-P N A}$. At $24 \mathrm{~h}$ after injection, the SUV for the tumor was $0.6 \pm 0.3$, whereas the tumor-to-blood and tumor-to-muscle activity concentration ratios were $0.5 \pm 0.1$ and $8 \pm 1$, respectively. At the same time point, however, elevated uptake was also observed in the kidneys, liver, and blood compared with control experiments, results attributed to the hybridization of the ${ }^{99 \mathrm{~m}} \mathrm{Tc}-\mathrm{PNA}$ with PNA-bearing immunoconjugate in the blood.

Affibody molecules (Affibody AB) —engineered proteins based on a 58 -amino-acid $(6.5 \mathrm{kDa})$ tri-helical scaffoldhave also proven to be effective radiopharmaceutical vectors both in mice and in humans, producing high-contrast images only hours after administration (28). The high renal reabsorption of Affibody molecules, however, presents a substantial obstacle to using directly labeled variants for targeted radiotherapy. Not surprisingly, in vivo pretargeting offers an opportunity to remedy this issue. To this end, Westerlund et al. created an anti-human epidermal growth factor receptor 2 (HER2) Affibody molecule bearing a 15-mer
PNA ( $\mathrm{Z}_{\mathrm{HER} 2}$-HP1) as well as a complementary PEGylated 15-mer PNA (HP2) that demonstrated fast hybridization, slow dissociation, rapid clearance from the blood, and low retention in healthy tissues (29). Honarvar et al. demonstrated the efficacy of Affibody molecule-based pretargeting using $\mathrm{Z}_{\mathrm{HER} 2}-\mathrm{HP} 1$ and ${ }^{111} \mathrm{In}$-labeled HP2 in mice bearing HER2expressing SKOV3 ovarian cancer xenografts (Fig. 4C) (30). The pretargeting strategy produced tumoral uptake of $19 \pm 2 \% \mathrm{ID} / \mathrm{g} 1 \mathrm{~h}$ after injection along with a $54 \pm 19$ tumorto-blood activity concentration ratio at the same time point. Critically, activity concentrations in the kidneys were about 50 times lower than those observed in experiments using directly labeled Affibody molecules, a development that the authors believe will enable pretargeted radiotherapy.

\section{APPROACH 4: CLICK CHEMISTRY}

Since the advent of click chemistry over a decade and a half ago, the drive to apply these selective transformations to in vivo ligations has remained strong. In this regard, the development of bioorthogonal click reactions-most notably the Staudinger ligation and the strain-promoted azidealkyne cycloaddition-proved critical, enabling selective chemistry within the complex environment of living systems. However, the sluggish kinetics of this first generation of bioorthogonal reactions made them unsuitable for in vivo pretargeting. This issue was largely solved in 2008 by the Fox Laboratory's resurrection of the IEDDA cycloaddition reaction between tetrazine and trans-cyclooctene (TCO; Fig. 5A). The IEDDA reaction is selective, modular, and bioorthogonal, but what really sets it apart from other click ligations is its speed. Rate constants for the reaction between tetrazine dienes and TCO dienophiles can exceed $100,000 \mathrm{M}^{-1} \mathrm{~s}^{-1}$, orders of magnitude faster than either the Staudinger or strain-promoted azide-alkyne cycloaddition ligations. The potential of the IEDDA reaction as a tool for bioconjugation was recognized almost immediately, and the ligation quickly found wide-sweeping applications in a variety of fields, including radiopharmaceutical chemistry (31).

The rapidity and bioorthogonality of the IEDDA reaction make it almost ideally suited for in vivo pretargeting (Fig. 5B), and perhaps not surprisingly, only 2 y passed before the first report of IEDDA-based pretargeting (32). In this pioneering work, the group of Rossin and Robillard used an anti-tumor-associated glycoprotein 72-targeting CC49-TCO immunoconjugate and an ${ }^{111}$ In-DOTA-labeled dipyridyltetrazine radioligand (Fig. 5C). SPECT imaging and biodistribution experiments revealed $4.2 \% \mathrm{ID} / \mathrm{g}$ in the tumor and a 13.1 tumor-to-muscle activity concentration ratio at $24 \mathrm{~h}$ after the injection of the radioligand, modest yet promising results that the authors later improved on via the development of a tetrazinebearing clearing agent (33). Notably, in this work-and almost all reports of IEDDA-based pretargeting - the TCO is attached to the antibody, and the tetrazine forms part of the radioligand, a choice that stems from the superior in vivo 


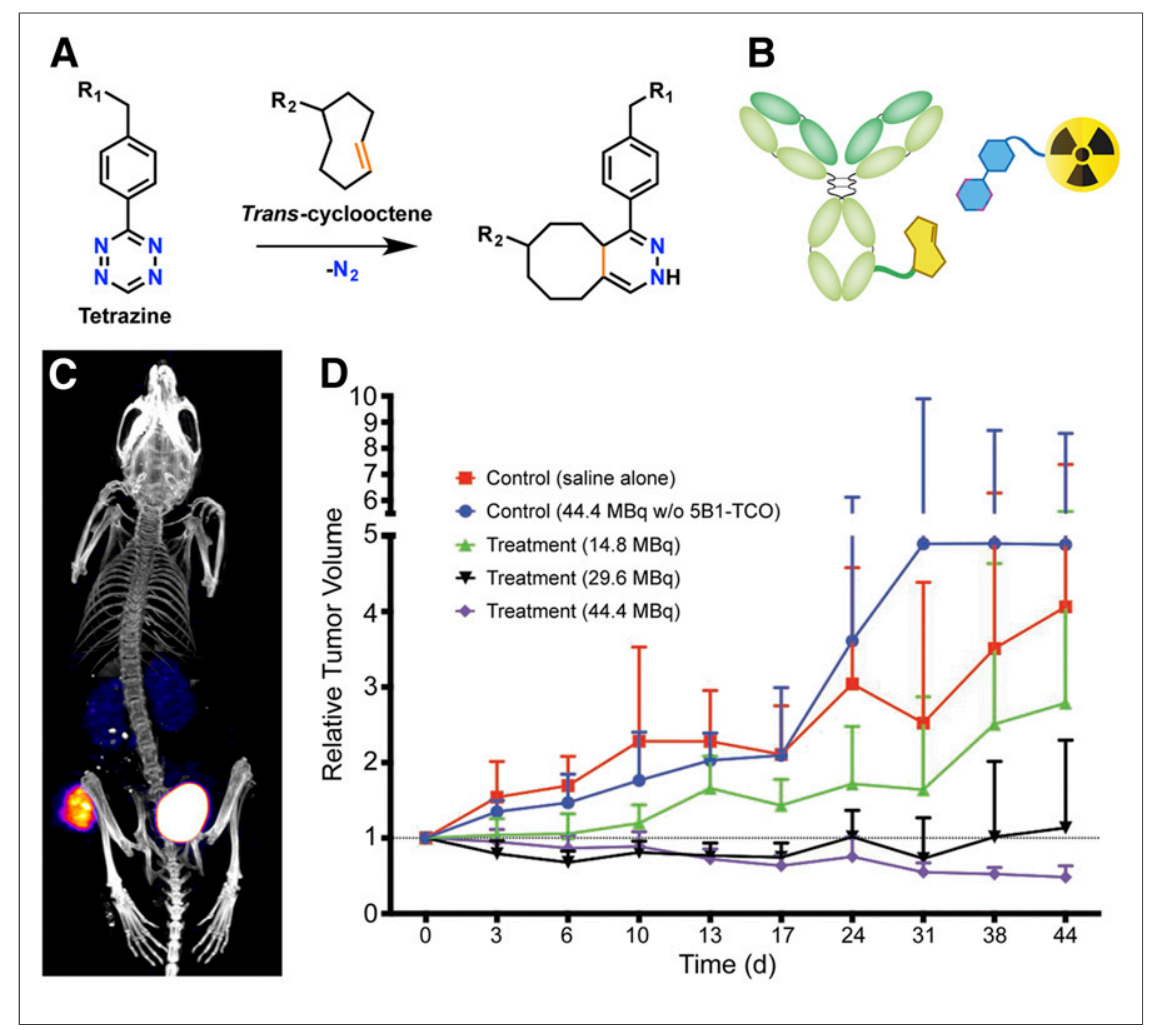

FIGURE 5. The IEDDA ligation (A); the 2 components of IEDDA-based pretargeting system: a TCO-bearing immunoconjugate and a tetrazine-modified radioligand $(\mathrm{B})$; SPECT/CT image of an LS174T tumor-bearing mouse pretargeted with CC49-TCO and ${ }^{111} \mathrm{In}$-DOTA-tetrazine (reprinted with permission of reference (34)) (C); and a longitudinal study of normalized tumor volume in mice bearing BXPC3 pancreatic ductal adenocarcinoma xenografts treated with a PRIT regimen composed of 5B1TCO and ${ }^{177}$ Lu-DOTA-PEG -tetrazine (reprinted with permission of (41)) (D).

that Houghton et al. first demonstrated the efficacy of this approach with a longitudinal therapy study (41). In this work, PRIT of mice bearing human pancreatic ductal adenocarcinoma xenografts using a CA19.9-targeting 5B1-TCO immunoconjugate and a ${ }^{177} \mathrm{Lu}$-DOTA-labeled tetrazine produced a dose-dependent therapeutic effect that all but completely eliminated tumor tissue at higher activities of the radioligand (Fig. 5D).

\section{CONCLUSION}

Reviews such as these often culminate in a critical comparison of the various strategies at hand. Unfortunately, however, contrasting the 4 approaches that we have discussed proves difficult for 2 reasons. First, the 4 methodologies have not been subject to a head-to-head competition using a single model system, rendering quantitative comparisons dubious at best. And second, each of the strategies stands at a different stage in its scientific development, a fact that inevitably muddies the waters of the conversation. Still, each approach clearly has its own set of advantages and disadvantages. Streptavidin-based strategies benefit

stability of the former. The group of Rossin and Robillard has since remained a leader in the field, extending its explorations to alternative TCO moieties and an IEDDA-activated therapeutic modality termed "click-to-release" (34).

Pretargeted PET was first reported by the laboratories of Weissleder and Lewis (35). Their approach used a colorectal cancer-targeting huA33-TCO immunoconjugate and a ${ }^{64} \mathrm{Cu}$-labeled tetrazine to produce high-contrast PET images at only a fraction of the radiation dose to healthy organs produced by directly labeled radioimmunoconjugates. The success of this work led others to investigate the use of even shorter-lived isotopes, including ${ }^{18} \mathrm{~F},{ }^{68} \mathrm{Ga}$, and ${ }^{11} \mathrm{C}(36,37)$. In recent years, several laboratories have begun investigating pretargeting with more rapidly clearing vectors-including TCO-bearing diabodies, bisphosphonates, and Affibody molecules - a shift that may enable same-day procedures and further improve the dosimetric advantages of the approach $(38-40)$.

PRIT using the tetrazine-TCO ligation has admittedly received less attention than imaging, yet it arguably holds even more promise. In 2013, Rossin et al. first provided biodistribution and dosimetry data to support the feasibility of tetrazine-TCO PRIT (33). However, it was not until last year from the multivalency of streptavidin as well as the remarkable affinity between the protein and biotin, yet both the immunogenicity of streptavidin and the presence of endogenous biotin have proven complicating factors. bsAbs have produced some of the finest clinical results to date, and the advent of HSG-based haptens eliminates some (but not all) of the concerns surrounding the modularity of these systems. Nonetheless, the complexity and expense of vector production still require attention. Oligonucleotide-centered approaches have produced some enticing preclinical results, but the sequence-dependent nature of hybridization rates and affinities as well as the hydrophobicity of PNAs must both be carefully considered as this technology evolves. Finally, strategies predicated on the IEDDA reaction have proven extremely effective; represent the only approaches that use covalent chemistry; and are modular, tunable, and completely bioorthogonal. However, some latent concerns remain surrounding the in vivo stability of the TCO and tetrazine moieties.

In the end, we believe that it is an incredibly exciting time for in vivo pretargeting, yet this excitement must be tempered somewhat by the exigencies of translational research. Three of the 4 strategies we have discussed face pivotal days in the 
near future. The approaches based on the IEDDA ligation and the hybridization of oligonucleotides both stand at a critical moment in their history: the move from the laboratory to the clinic. In contrast, pretargeting based on bsAbs has produced extremely promising clinical results; however, the modularity and complexity of these vectors remain concerns going forward. Moving beyond these established methodologies, there is equally fascinating work to be done unearthing the next generation of pretargeting strategies, whether they are predicated on host-guest relationships, enzymatic transformations, biologic binding partners, or yet-to-be-discovered bioorthogonal reactions. On all of these counts, we look forward with optimism to the data and developments of the years to come.

\section{DISCLOSURE}

This work is supported by the NIH (4R00CA178205-02), NIHMD (G12MD007599), TeamConnor Childhood Cancer Foundation, the Swedish Cancer Society (CAN 2015/350), Hunter College, and Uppsala University. No other potential conflict of interest relevant to this article was reported.

\section{REFERENCES}

1. Reardan DT, Meares CF, Goodwin DA, et al. Antibodies against metal chelates. Nature. 1985;316:265-268.

2. Hnatowich DJ, Virzi F, Rusckowski M. Investigations of avidin and biotin for imaging applications. J Nucl Med. 1987;28:1294-1302.

3. Goldenberg DM, Chang CH, Rossi EA, McBride WJ, Sharkey RM. Pretargeted molecular imaging and radioimmunotherapy. Theranostics. 2012;2:523-540.

4. van de Watering FC, Rijpkema M, Robillard M, Oyen WJ, Boerman OC. Pretargeted imaging and radioimmunotherapy using antibodies and bioorthogonal chemistry. Front Med. 2014;1:44.

5. Casalini P, Luison E, Menard S, Colnaghi MI, Paganelli G, Canevari S. Tumor pretargeting: role of avidin/streptavidin on monoclonal antibody internalization. J Nucl Med. 1997;38:1378-1381.

6. Sung C, van Osdol WW. Pharmacokinetic comparison of direct antibody targeting with pretargeting protocols based on streptavidin-biotin binding. $\mathrm{J} \mathrm{Nucl} \mathrm{Med}$. 1995;36:867-876.

7. Kalofonos HP, Rusckowski M, Siebecker DA, et al. Imaging of tumor in patients with indium-111-labeled biotin and streptavidin-conjugated antibodies: preliminary communication. J Nucl Med. 1990;31:1791-1796.

8. Weiden PL, Breitz HB. Pretargeted radioimmunotherapy for treatment of nonHodgkin's lymphoma. Crit Rev Oncol Hematol. 2001;40:37-51.

9. Grana C, Chinol M, Robertson C, et al. Pretargeted adjuvant radioimmunotherapy with yttrium-90-biotin in malignant glioma patients: a pilot study. Br J Cancer. 2002;86:207-212.

10. Forero A, Weiden PL, Vose JM, et al. Phase 1 trial of a novel anti-CD20 fusion protein in pretargeted radioimmunotherapy for B-cell non-Hodgkin lymphoma. Blood. 2004;104:227-236.

11. Cheal SM, Xu H, Guo HF, Zanzonico PB, Larson SM, Cheung NK. Preclinical evaluation of multistep targeting of diasialoganglioside GD2 using an IgG-scFv bispecific antibody with high affinity for GD2 and DOTA metal complex. Mol Cancer Ther. 2014;13:1803-1812.

12. Orcutt KD, Slusarczyk AL, Cieslewicz M, et al. Engineering an antibody with picomolar affinity to DOTA chelates of multiple radionuclides for pretargeted radioimmunotherapy and imaging. Nucl Med Biol. 2011;38:223-233.

13. de Boisferon MH, Manetti C, Raguin O, et al. Pretargeted radioimmunotherapy using I-131-labelled bivalent hapten-bearing peptides. Lett Pept Sci. 1997;4:331339.

14. Gautherot E, Rouvier E, Daniel L, et al. Pretargeted radioimmunotherapy of human colorectal xenografts with bispecific antibody and I-131-labeled bivalent hapten. J Nucl Med. 2000;41:480-487.

15. Boerman OC, Kranenborg MHGC, Oosterwijk E, et al. Pretargeting of renal cell carcinoma: improved tumor targeting with a bivalent chelate. Cancer Res. 1999;59:4400-4405.

16. Kraeber-Bodéré F, Rousseau C, Bodet-Milin C, et al. Targeting, toxicity, and efficacy of 2-step, pretargeted radioimmunotherapy using a chimeric bispecific antibody and I-131-labeled bivalent hapten in a phase I optimization clinical trial. J Nucl Med. 2006;47:247-255.

17. Rossi EA, Goldenberg DM, Cardillo TM, McBride WJ, Sharkey RM, Chang $\mathrm{C}-\mathrm{H}$. Stably tethered multifunctional structures of defined composition made by the dock and lock method for use in cancer imaging. Proc Natl Acad Sci USA. 2006;103:6841-6846.

18. van Rij CM, Frielink C, Goldenberg DM, et al. Pretargeted radioimmunotherapy of prostate cancer with an anti-TROP-2xAnti-HSG bispecific antibody and a Lu177-labeled peptide. Cancer Biother Radiopharm. 2014;29:323-329.

19. Sharkey RM, van Rij CM, Karacay H, et al. A new Tri-Fab bispecific antibody for pretargeting Trop-2-expressing epithelial cancers. J Nucl Med. 2012;53: 1625-1632.

20. Schoffelen R, Boerman OC, Goldenberg DM, et al. Development of an imagingguided CEA-pretargeted radionuclide treatment of advanced colorectal cancer: first clinical results. Br J Cancer. 2013;109:934-942.

21. Bodet-Milin C, Faivre-Chauvet A, Carlier T, et al. Immuno-PET using anticarcinoembryonic antigen bispecific antibody and Ga-68-labeled peptide in metastatic medullary thyroid carcinoma: clinical optimization of the pretargeting parameters in a first-in-human trial. J Nucl Med. 2016;57:1505-1511.

22. Kraeber-Bodéré $\mathrm{F}$, Rousseau $\mathrm{C}$, Bodet-Milin $\mathrm{C}$, et al. A pretargeting system for tumor PET imaging and radioimmunotherapy. Front Pharmacol. 2015;6:54.

23. Liu G, Mang'era K, Liu N, Gupta S, Rusckowski M, Hnatowich DJ. Tumor pretargeting in mice using Tc-99m-labeled morpholino, a DNA analog. $\mathrm{J} \mathrm{Nucl}$ Med. 2002;43:384-391.

24. Liu G, Dou SP, Liu YX, Wang YZ, Rusckowski M, Hnatowich DJ. Y-90 labeled phosphorodiamidate morpholino oligomer for pretargeting radiotherapy. Bioconjug Chem. 2011;22:2539-2545.

25. He J, Wang Y, Dou SP, et al. Affinity enhancement pretargeting: synthesis and testing of a Tc-99m-labeled bivalent MORF. Mol Pharm. 2010;7:1118-1124.

26. Liu G, Dou S, Cheng D, et al. Human islet cell MORF/cMORF pretargeting in a xenogeneic murine transplant model. Mol Pharm. 2011;8:767-773.

27. Leonidova A, Foerster C, Zarschler $\mathrm{K}$, et al. In vivo demonstration of an active tumor pretargeting approach with peptide nucleic acid bioconjugates as complementary system. Chem Sci. 2015;6:5601-5616.

28. Sörensen J, Velikyan I, Sandberg D, et al. Measuring HER2-receptor expression in metastatic breast cancer using Ga-68 ABY-025 affibody PET/CT. Theranostics. 2016;6:262-271.

29. Westerlund K, Honarvar H, Tolmachev V, Karlstrom AE. Design, preparation, and characterization of PNA-based hybridization probes for affibody-moleculemediated pretargeting. Bioconjug Chem. 2015;26:1724-1736.

30. Honarvar H, Westerlund K, Altai M, et al. Feasibility of affibody molecule-based PNA-mediated radionuclide pretargeting of malignant tumors. Theranostics. 2016;6:93-103.

31. Meyer J-P, Adumeau P, Lewis JS, Zeglis BM. Click chemistry and radiochemistry: the first 10 years. Bioconjug Chem. 2016;27:2791-2807.

32. Rossin R, Renart Verkerk P, van den Bosch SM, et al. In vivo chemistry for pretargeted tumor imaging in live mice. Angew Chem Int Ed Engl. 2010;49:33753378.

33. Rossin R, Lappchen T, van den Bosch SM, Laforest R, Robillard MS. Diels-Alder reaction for tumor pretargeting: in vivo chemistry can boost tumor radiation dose compared with directly labeled antibody. J Nucl Med. 2013;54:1989-1995.

34. Rossin R, van Duijnhoven SMJ, Läppchen T, van den Bosch SM, Robillard MS. Trans-cyclooctene tag with improved properties for tumor pretargeting with the Diels-Alder reaction. Mol Pharm. 2014;11:3090-3096.

35. Zeglis BM, Sevak KK, Reiner T, et al. A pretargeted PET imaging strategy based on bioorthogonal Diels-Alder click chemistry. J Nucl Med. 2013;54:1389-1396.

36. Denk C, Svatunek D, Mairinger S, et al. Design, synthesis, and evaluation of a low-molecular-weight ${ }^{11} \mathrm{C}$-labeled tetrazine for pretargeted PET imaging applying bioorthogonal in vivo click chemistry. Bioconjug Chem. 2016;27: 1707-1712.

37. Meyer J-P, Houghton JL, Kozlowski P, et al. ${ }^{18} \mathrm{~F}$-based pretargeted PET imaging based on bioorthogonal Diels-Alder click chemistry. Bioconjug Chem. 2016;27:298301.

38. Yazdani A, Bilton H, Vito A, et al. A bone-seeking trans-cyclooctene for pretargeting and bioorthogonal chemistry: a proof of concept study using ${ }^{99 \mathrm{~m}}$ Tc- and ${ }^{177}$ Lu-labeled tetrazines. J Med Chem. 2016;59:9381-9389.

39. Altai M, Perols A, Tsourma M, et al. Feasibility of affibody-based bioorthogonal chemistry mediated radionuclide pretargeting. J Nucl Med. 2016;57:431-436.

40. van Duijnhoven SMJ, Rossin R, van den Bosch SM, Wheatcroft MP, Hudson PJ, Robillard MS. Diabody pretargeting with click chemistry in vivo. $J$ Nucl Med. 2015;56:1422-1428.

41. Houghton JL, Membreno R, Abdel-Atti D, et al. Establishment of the in vivo efficacy of pretargeted radioimmunotherapy utilizing inverse electron demand Diels-Alder click chemistry. Mol Cancer Ther. 2017;16:124-133. 